\title{
17
}

\section{Environmental Information for Public Access}

\author{
Peter Kutschera, Gerald Schimak, Heinrich Humer \\ All Austrian Research Centre Seibersdorf \\ Dept. of Information Technology \\ Kutschera@zdfzs.arcs.ac.at, Schimak@zdfzs.arcs.ac.at, \\ Humer@zdfzs.arcs.ac.at
}

\begin{abstract}
After the installation of the Austrian Ozone Monitoring Network in April 1994, it was soon clear that the information gathered all over the country should be provided to all interested in that matter. For that task an acceptable technology was found by using World Wide Web. To demonstrate how this new technology can be used to access actual environmental information via meta information the Austrian Research Centre Seibersdorf developed an WWW prototype.

In the following chapters a World Wide Web (WWW) gateway for the Austrian Ozone Monitoring Network will be described, first emphasizing some aspects of the Austrian Ozone Monitoring Network and second talking in detail about the implemented data presentation for environmental information.
\end{abstract}

\section{Keywords}

distributed environmental information system, air pollution monitoring system, visualization, integration, internet

\section{SNAPSHOT OF THE OZONE MONITORING SITUATION IN AUSTRIA}

At the moment there are about 180 measuring stations feeding continuously meteorological and pollutant information into the Austrian Ozone Monitoring Network. This stations integrated in local air quality measurement networks are operated by the responsible authorities in the nine Austrian federal countries. An additional tenth is operated by the Federal Environmental Agency Austria (FEAA) as nationwide background monitoring system.

To overcome the differences in the air quality measurement networks, the complexity of distributed data as well as heterogenity within the network the Austrian Research Centre Seibersdorf developed a solution to harmonize access to all of this stations within the network. The keywords for the new technology used are ozone network computer, 
meta information system, meta data distribution and integration of different environmental information. These methods are exhausted discussed in (Schimak 1994a and Schimak $1994 \mathrm{~b})$. Whenever there is a new or modified data in an air quality measurement network's central computer system the data is automatically transformed into the ozone data network format and sent over a local area network to the ozone network computer, where the values are stored in a local database. Thereafter the information is accessible by all remote ozone network computers. The measured, aggregated and stored values will be accessed by a high speed retrieval mechanism (e.g. formula server) installed on each network node. All ozone network computers are linked via public X.25 packet switched data network. Technical information about the server concept you will find in (Schimak 1994a and Schimak 1994b).

The continuously available information within the Austrian Ozone Network can be scaled in meta information and average values, like half hour mean values.

\subsection{Meta information}

Meta information can be described as information about information. It is a special kind of descriptive data and it is used in the Austrian Ozone Monitoring Network to maintain and update the network as well as to distribute and transform data. For example you would like to get information about the actual measured ozone concentration of a special monitoring area: So you use your local conventions for your query as you always did, without worrying about how your neighbor measured the values belonging to the requested monitoring area. The values are presented to you as you have always been familiar with. The system itself performs for you the hard work of transforming methods and data.

The following meta information is necessary to run the Austrian Ozone Monitoring Network:

1. the operator of the local air quality measurement systems

2. the measuring stations and

3. measured components (primary and secondary pollutant, meteorological parameters, etc..)

4. formulas, averages, units and thresholds

These main categories of meta information in the Austrian Ozone Monitoring System everyone can easily retrieve by using different tools (see below).

\subsection{Measured values}

The measured values of one component are aggregated to and stored as half hour mean values in an environmental database. Each of these values is described by additional attributes (flags) providing information about validity and check state. Higher aggregated averages can be calculated by doing some simple definitions in the meta database.

Access to values as well as to meta information is organized by using remote procedure calls. Different implemented retrieval mechanism realize the transformation from local or remote access methods and spelling conventions into a global one. Such mechanisms exist e.g. for time series retrieval (implemented within the formula server), one for meta data 
updates and distribution (implemented within the meta data server), one for communication (implemented within the ozone network server), etc...

The Austrian Ozone Monitoring Network Application consists of a set of different tools for the daily work at the local authorities. These tools (discussed in (Schimak 1994c)) allow to generate daily ozone monitoring reports, to analyze time series and to visualize the current ozone situation as well as to support the handling of the austrian ozone alarming scheme.

\section{THE WWW (WORLD WIDE WEB) GATEWAY}

As the public interest was rapidly growing, knowing to have now valid information about the current ozone situation in Austria accessible, the Austrian Research Centre Seibersdorf in cooperation with the FEAA forced the intention to develop a WWW prototype to demonstrate how environmental information can be electronically presented to the public.

In fall 1994, after the end of the first ozone period, as it was stated that the software system is capable to work under pressure, a first release of a WWW prototype was installed at the Austrian Research Centre.

\subsection{What is WWW}

WWW [1, 2] (Weichselgartner, 1993 and Klute, 1994) is a new method to harmonize the different Internet services. Based on hypermedia documents and gateways for News, Gopher, Archie and FTP it was tried to simplify data retrieval in distributed systems. WWW consists of a large set of worldwide distributed hypertext documents offered by many WWW server. Each of these documents may have links to further documents or gateways for further Internet services and so on.

Access to WWW is usually performed by WWW clients. Under X Windows Mosaic is the most common user interface for WWW interactions, perhaps there are also Clients for Windows, Mac or even ASCII Terminals available. Clients providing a user interface have the great advantage that the user may not care about links. He only click on an underlined item in the document and gets data (spread all over the world) presented as he would have them locally. In terms of the Austrian Ozone Monitoring Network it means that he gets information from several air quality measurement networks.

\subsection{Accessible information}

In general public access (all around the world) is provided via the Seibersdorf -WWWgateway. The server prototype itself distinguishes where the requests come from (authorized users, others) and filters the requested data depending on a special identification mechanism. The information you can retrieve is mostly the same as stored within the Austrian Ozone Monitoring Network and discussed above.

Therefore you are able to retrieve all meta data about measuring stations (list of stations) inclusive the description of each measuring station, coordinates, list of measured components as well as measured values (only checked values)

The provided information is in no way restricted to the user (accept the above mentioned privileges) thus in case of special treated data (e.g. calibration occurred during the 
measured period) confusion can be caused by the observer not familiar with air quality data.

Monitoring information is presented in an interactive way giving the user the ability to decide how data will be visualized. In all this presentations (Figure 1 - Figure 4) only checked and validated data are used to display ozone or meteorological data.

One of the great advantage of WWW compared with file access (FTP) is that it is possible to generate the information presentation on demand as there exists to many possibilities to offer all presentations pre-formatted. The disadvantage is, that the fetching and preparing of the requested presentation may consume time. So it gets impossible to generate for example animations (mpeg videos) on demand. There you will currently reach the upper limit of the involved computer technology. But it is possible to generate some videos in advance and give access by the WWW.

\subsection{The WWW user interface}

The user interface consists of some WWW pages. These pages are generated when the Seibersdorf WWW server gets a request via the gateway. The start page of the gateway to the Austria Ozone Monitoring Network is driven by the following URL (Berners-Lee, 1993)

http://zditr1.arcs.ac.at/odv-bin/data/ui.pl

The server is capable to decide where the request comes from, and presents the start page in german or english.

\section{Presentation of ozone monitoring information}

After having read the general information about the project you can select the kind of data presentation you are familiar with:

- two dimensional,

- three dimensional,

- colored two dimensional,

- beams about map,

- colors about map,

- mpeg-files (animations, videos)

The links two dimensional, three dimensional and with colors points to the map of Austria (figure 1) where you can interactively select a component from a list of pollutants and meteorological parameters as well as a time interval (for example one day / one week / one month) and an ozone monitoring station. When a station is selected by simply clicking with the mouse onto a square (representing the ozone monitoring station) within the map the values are automatically retrieved from the Austrian Ozone Monitoring Network and transformed to the requested presentation. When no station is armed with the mouse pointer (clicking between the stations) a list of stations sorted by distance from the mouse pointer is displayed to the user, where he may select stations by name.

The link Beams (meters) about map refers to a selection of one or more components. Selecting this presentation type the actually needed values are fetched simultaneously from 


\section{Measuring stations in Austria}

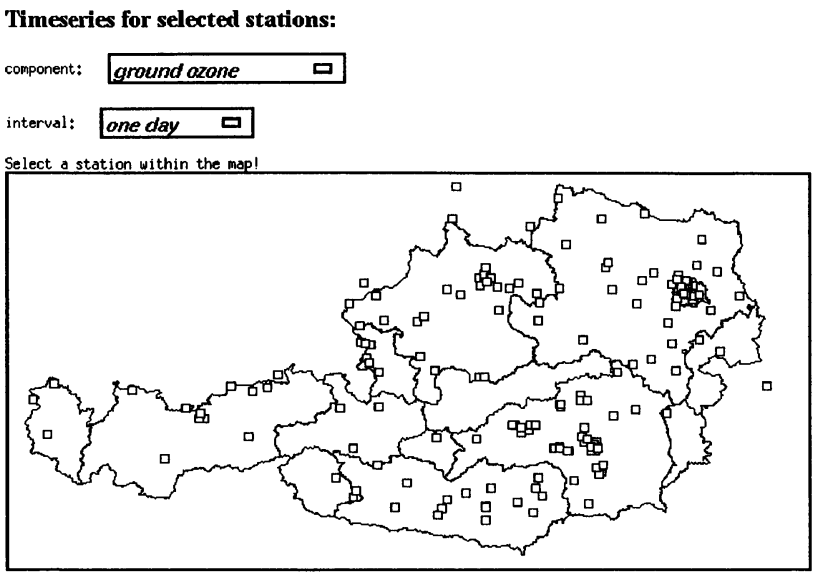

Peter Kutschera, 31. 5. 1994, öfZS

Figure 1 Selection of a monitoring station

distinct servers across the network. As there are all (10 at the moment) ozone monitoring network servers involved and information from every measuring station within the network is fetched this may cause some delay, depending on network overload.

The last link on the start page refers to a directory containing pre-calculated animations (mpeg videos).

Two dimensional data presentation: In figure 2 the two dimensional presentation of time series of one component and one station is displayed. This is the only data presentation where you are allowed to interpret single values. All the other presentations show only an overview to the trends.

There is a blue line (threshold) in the graph, which can't be seen in the hardcopy presenting the value $200 p p b$. Above this value some actions (alarm scheme) will be carried out by the Austrian government. At the end of the month $(27 t h, 28 t h)$ you can see the typical up and down of ground ozone values within on day.

If slight changes occur from one day to the other this can't be analyzed with this kind of data presentation. Therefore use the data prescntations described in the next paragraphs.

Three dimensional data presentation: In figure 3 the three dimensional presentation of a time series of one component and one station is shown. The hours within one day are drawn from left to right to show the changes of the values within one day. On the left side the days are drawn to show the changes from one day to the next. 
Values from Illmitz (ground ozone)

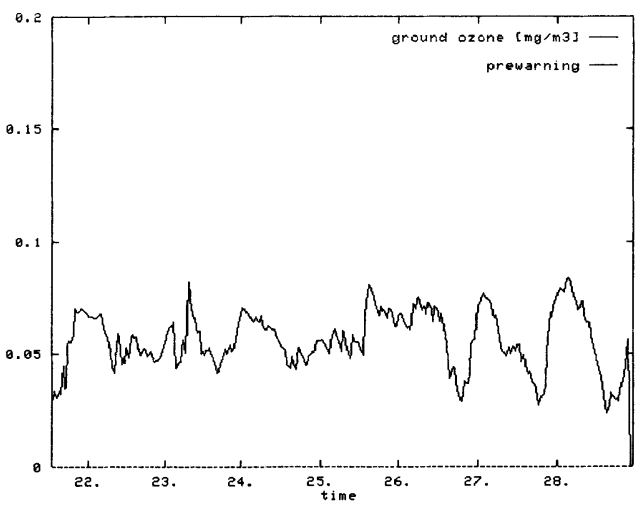

Figure 2 two dimensional presentation of time series

\section{Values from Illmitz (ground ozone)}

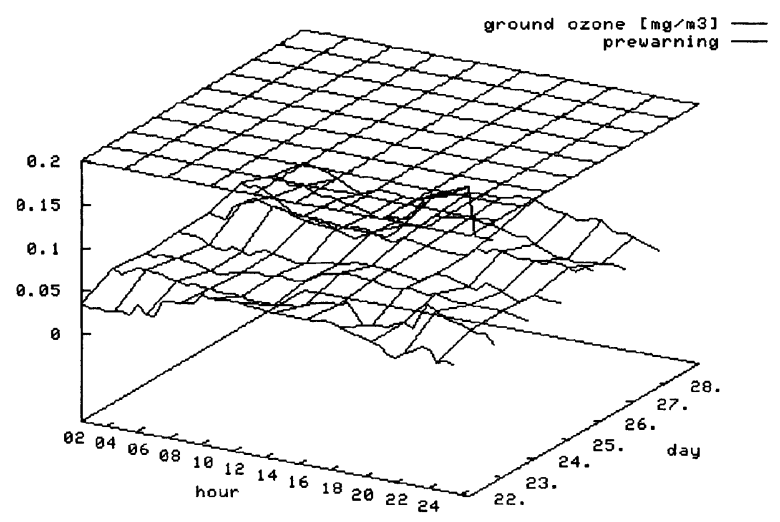

Figure 3 three dimensional presentation of time series 


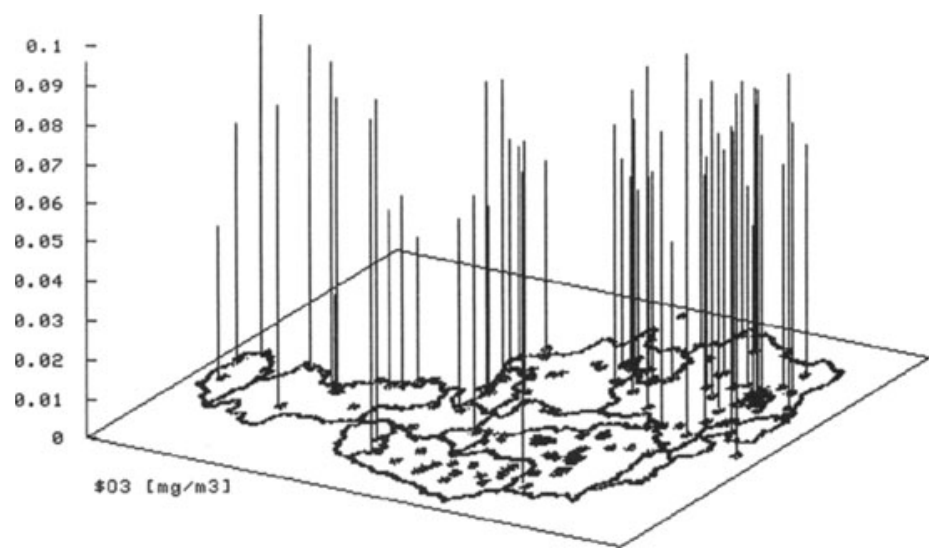

Figure 4 Beams about map

Colored data presentation: This presentation is more or less equal to the three dimensional presentation. Hours within one day are displayed from left to right to show the changes of the values within one day. Days are scaled from top to bottom.

The values are color-indicated. Its a kind of data presentation that lets you really well recognize daily (like rush hours in the morning, etc...) or long term trends (distribution of a pollutant concentration during an ozone period).

Beams about map: After selecting one or more components the figure 4 is shown. Here the values for one component (more would be possible) of all currently reachable stations are shown, but only for one time stamp. The values are shown as beams. It's not possible to get the exact values from this picture but it is useful to get a quick overview about the current ozone situation.

Animations (Mpeg videos): At the moment some pre-calculated mpeg videos can be accessed, but it is really difficult to make a hardcopy. So please have a look to our WWW pages.

\subsection{Brief technical details}

The WWW gateway was implemented using perl (Wall 1991 and Wall 1993) scripts. Perl is a special kind of script language, which is nearly available on each operating system. Graphical output was generated using "gnuplot" and "ppm+".

The Austrian Ozone Network Server is currently installed on a DECStation 5000/125. The operating system is ULTRIX. Remote as well as local data access is done by RPCs (remote procedure calls). 


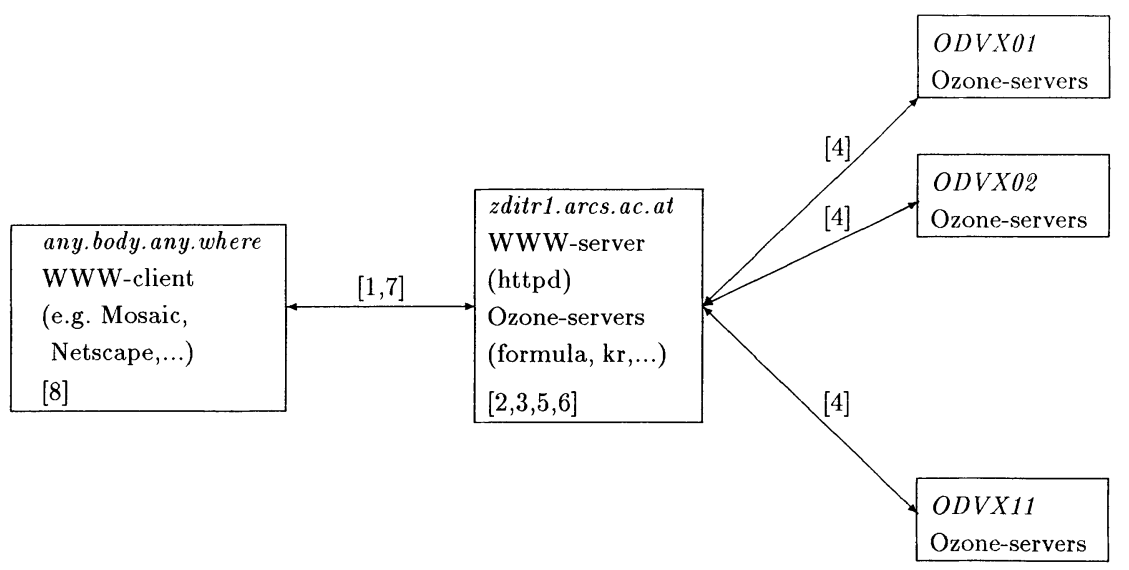

Figure 5 Server and clients

Figure 5 demonstrates the involved client and server applications used to retrieve actual ozone information. The numbers within figure 5 indicates the following actions:

1. WWW client requests URL from WWW server

2. WWW server (httpd) calls script (perl)

3 . script queries information from local ozone server

4. local ozone server queries measured data from the other ozone servers in the Austrian Ozone Measurement Network

5. script generates picture (gif)

6. script generates result page (html)

7. WWW server sends result page

8. WWW client displays result

The servers in the ozone network are described in (Schimak 1994a).

The generation of the pictures, especially if more than one ozone server is involved, needs some minutes since most of the servers can only be reached which 2400bps. If other communication takes place it might take a little bit longer.

\section{FUTURE ASPECTS}

Beside the yet existing data presentation possibilities shown in figure 1 up to figure 4 , for the future the following domains should be graphically displayed (Schimak 1994c):

1. ozone alarm 
2. different graphical data presentation of time series for a single component and measuring station

3. combination of several components.

4. different presentations (numbers, colors, beams) of values from all stations in a map (no time series).

5 . interpolated ozone values about the austrian area.

6 . the information from 4 and 5 as animation

Currently a simulation and interpolation model is integrated into the Austrian Ozone Monitoring System. A future aspect may be to link the output of this model to the WWW application to present a high sophisticated model to the public.

\section{CONCLUSION}

The gateway to the WWW can be used to give many people access to the environmental information. We could demonstrate that the realization of a usable prototype is easy and cheap and that the WWW is a practical tool to provide environmental information as well as to develop simple front-end visualization applications.

\section{BIOGRAPHY}

Information about the authors can be found on

http://zditr1.arcs.ac.at/ peter

http://zditr1.arcs.ac.at/ schimak

http://zditr1.arcs.ac.at/ ${ }^{\sim}$ humer

\section{REFERENCES}

Erich Weichselgartner (1993) Unendliche Tiefen: WWW: Hypertext-basiertes Informationswerkzeug im Internet, iX 12/93, S. 84ff.

Rainer Klute (1994) Zusammengewebt: Internet-Informationsdienste mit Mosaic, iX 2/94, S. $150 \mathrm{ff}$.

Tim Berners-Lee (1993): Uniform Resource Locators; A Unifying Syntax for the Expression of Names and Addresses of Objects in the Network, Internet Draft, 14. October 1993,

ftp://info.cern.ch/pub/www/doc/url7a.ps

Schimak G.(1994a), Denzer R., Humer H.: Integration in the Austrian Ozone Network, 2nd Workshop on the Integration of Environmental Data (Integration von Umweltdaten), Dagstuhl '94, R.Guettler and W.Geiger (eds.), Metropolis Verlag, Marburg

Schimak G.(1994b), Humer H., Denzer R., Knappitsch E.: The Ozone Network for Austria: Technical Concept of a Distributed Environmental Information System, 8. Symposium Informatik fuer den Umweltschutz - Umweltinformatik 94, L. M. Hilty, A. Jaeschke, B. Page and A. Schwabl (eds.), Hamburg 94, Metropolis-Verlag, Marburg 
Schimak G. (1994c), Denzer R., Humer H.: Interactive Visualization in the Austrian Ozone Network, 4. Workshop, Visualization of Environmental Data (Visualisierung von Umweltdaten), Dagstuhl '94, R. Denzer, R.Guettler and H. Deutsch (eds.), Metropolis Verlag, Marburg

Wall L. (1991) \& Schwartz R. L.: Programming perl, O'Reilly \& Associates, 1991

Wall L. (1993)\& Schwartz R. L.: Learning perl, O'Reilly \& Associates, 1993 